\title{
Tratamiento quirúrgico de la gangrena de Fournier
}

\author{
Wilfredo Calderón O. ${ }^{1,2}$, Juan Pablo CamachoM. ${ }^{1,2}$, Miguel Obaíd G. ${ }^{1,2}$, \\ Javier Moraga C. ${ }^{1,2}$, David Bravo L. ${ }^{1,3}$ y Daniel Calderón M.,
}

'Facultad de Medicina Universidad de Chile. Santiago, Chile. ${ }^{2}$ Servicio de Cirugía Hospital del Salvador. Santiago, Chile. 3Servicio de Cirugía Hospital San Juan de Dios. Santiago, Chile.

${ }^{4}$ Servicio de Cirugía Hospital Luis Tisné Brousse. Santiago, Chile.

Recibido 2020-01-06 y aceptado 2020-03-03

Correspondencia a: Dr. Wilfredo Calderón Orteg wilfredocalder@gmail.com

\section{Surgical management of Fournier's gangrene}

Aim: To describe the surgical management performed for genital-perineal reconstruction (GPR) in patients with sequelae of Fournier gangrene (FG). Materials and Method: It is based on a retroactive series of cases of patients with effects of FG who were given GPR between 2011 and 2019. We performed a descriptive analysis using the variables surgical technique, age, sex, comorbidities, anatomical subunits affected, anatomic origin of the Fournier's gangrene, number of surgical procedures, colostomy procedure, negative pressure therapy, Flexi-Seal ${ }^{\circledR}$, bacteria isolated, hospital stay, type of reconstructive procedures and complications. We performed GPR on 43 patients (81.1\% male), with a mean age of 59.1 (17-86 years); 72\% were diabetic. The number of subunits involved was directly and significantly associated with the number of surgical interventions. Results: The reconstruction techniques most used were, in descending order: flaps $(23.2 \%)$, wound closure and split-thickness skin graft (STSG) (23.2\%), primary closure $(16.2 \%)$, STSG (16.2\%), secondary closure (9.3\%), flap and STSG $(6.9 \%)$ and partial wound closure for healing of the remaining area for a second intention (4.6\%). Discussion: The reconstruction choice is based on the characteristics of the defect, that is, the size, location and depth, as well as the availability of local tissue. Preferably opt for primary closures without tension, followed by flaps and IPP. Conclusion: The RGP is a challenge for the plastic surgeon. The techniques described have proven safe and reproducible for the surgical treatment of Fournier Gangrene.

Key words: Fournier's gangrene; genitoperineal reconstruction; flaps.

\section{Resumen}

Objetivo: Describir el manejo quirúrgico realizado para la reconstrucción genitoperineal (RGP) en pacientes con secuelas de Gangrena de Fournier (GF). Materiales y Método: Corresponde a una serie de casos retrospectiva de pacientes con secuelas de GF a los que se les realizó RGP entre los años 2011 y 2019. Se realizó un análisis descriptivo con las variables de técnica quirúrgica, edad, sexo, comorbilidades, subunidades anatómicas afectadas, origen anatómico de la gangrena de Fournier, número de procedimientos quirúrgicos, procedimiento de colostomía, terapia de presión negativa, Flexi-Seal ${ }^{\circledR}$, bacterias aisladas, duración de estancia hospitalaria, tipo de procedimientos reconstructivos y complicaciones. Resultados: Se realizó RGP a 43 pacientes (81,1\% hombres), con un promedio de edad de 59,1 (17-86 años), 72,7\% eran diabéticos. El número de subunidades involucradas se asocia directamente y significativamente en relación al número de intervenciones quirúrgicas. Las técnicas utilizadas para la reconstrucción en orden de frecuencia fueron: colgajos $(23 \%)$, cierre parcial más injerto dermoepidérmico de grosor parcial (IPP) (20\%), cierre parcial (16\%) e IPP (16\%), cierre por segunda intención (10\%), colgajo más IPP (7\%) y cierre parcial para cierre por segunda intención de zona restante $(5 \%)$. Discusión: La elección de reconstrucción se basa en las características del defecto, es decir, el tamaño, la ubicación y profundidad, así como la disponibilidad de tejido local. De preferencia optar por cierres primarios sin tensión, seguido de colgajos y de IPP. Conclusión: La RGP es un desafío para el cirujano plástico. Las técnicas descritas han demostrado ser seguras y reproducibles para el tratamiento quirúrgico de la gangrena de Fournier.

Palabras clave: gangrena de Fournier; reconstrucción genitoperineal; colgajos. 


\section{Introducción}

La gangrena de Fournier (GF) es una forma rápidamente progresiva y potencialmente letal de fasceitis necrotizante de las regiones perineal, genital o perianal, que conduce trombosis de pequeños vasos subcutáneos y necrosis de la piel subyacente ${ }^{1,2}$. La inflamación y el edema causado por infección polimicrobiana, conducen una endarteritis obliterante de las arterias subcutáneas promoviendo la disección perifascial con diseminación bacteriana y progresión a gangrena del tejido subcutáneo y piel $^{3}$. La GF fue descrita por primera vez por Jean Alfred Fournier en $1883^{4,5}$ y es causada en su mayor parte por una infección sinérgica aerobia y anaerobia originada de un sitio de infección colorrectal, genitourinario o cutáneo en asociación con alguna forma de inmunosupresión subyacente ${ }^{6}$. Los factores de riesgos comunes de esta enfermedad incluyen edad avanzada, diabetes, alcoholismo, procesos oncológicos e inmunodeficiencia ${ }^{4}$. El reconocimiento temprano junto con una extensa resección del tejido necrótico, tratamiento antimicrobiano rápido $\mathrm{y}$ adecuado, $\mathrm{y}$ volemización agresiva, son el pilar del manejo de la gangrena de Fournier, logrando reducir la morbilidad y la mortalidad en estos pacientes?

A pesar del manejo avanzado, la mortalidad sigue siendo elevada, $3 \%-67 \%$, dependiendo de las series ${ }^{8}$. Después del desbridamiento quirúrgico inicial, se requiere habitualmente de varios aseos quirúrgicos ${ }^{9,10}$ que originan en muchos pacientes significativos defectos de piel y partes blandas necesitando de la cirugía reconstructiva para lograr una adecuada cobertura, resultados funcionales y cosméticos satisfactorios ${ }^{11}$. Dentro de las técnicas de reconstrucción utilizadas para estos fines se encuentran: cierre primario, cierre por segunda intención, injertos de piel parcial (IPP), colgajos y la combinación de las anteriores.

El objetivo de este estudio es comunicar los resultados quirúrgicos observados para la reconstrucción genitoperineal (RGP) de una serie de pacientes con secuelas de GF, por el equipo de Cirugía Plástica del Hospital del Salvador (HS), entre los años 2011 y 2019.

\section{Materiales y Método}

Corresponde a un estudio retrospectivo y descriptivo de pacientes ingresados al Servicio de Cirugía Plástica de los Hospitales del Salvador y Luis Tisné Brousse, de Santiago de Chile, a partir de junio de 2011 hasta junio de 2019. La muestra corresponde a pacientes con diagnóstico de gangrena de Fournier que fueron sometidos a resección genitoperineal e ingresaron al servicio de cirugía plástica para manejo médico y quirúrgico. Se registraron las variables de edad, sexo, comorbilidades, subunidades afectadas, origen anatómico de la gangrena de Fournier, número de procedimientos quirúrgicos, procedimiento de colostomía, terapia de presión negativa, Flexi-Seal ${ }^{\circledR}$, bacterias aisladas, duración de estancia hospitalaria, tipo de procedimientos reconstructivos y complicaciones.

Inicialmente se administraron antibióticos de amplio espectro y luego se ajustaron según el antibiograma del primer cultivo cualitativo y cuantitativo de tejido tomado luego del primer aseo quirúrgico en pabellón. Se realizaron todos los aseos quirúrgicos necesarios hasta lograr un lecho limpio con tejido granulatorio, en ausencia de tejido esfacelado o necrótico. Finalmente, se programó para cobertura del defecto dependiendo del tamaño del defecto y subunidad involucrada: periné anterior, periné posterior, inguinal, glúteo, escroto, vulvar, pene, extremidad inferior (muslo), pubis, abdomen. Pacientes con ano flotante o lesión extensa perianal de difícil control de contaminación por las heces, se indicó Flexi-Seal ${ }^{\circledR}$ o colostomía en asa laparoscópica. Indistintamente en algunos pacientes se les indicó uso de terapia de presión negativa $\left(\mathrm{VAC}^{\circledR}\right)$. Dependiendo de las subunidades involucradas y extensión, se realizaron distintas técnicas de cobertura: cierre primario, injertos dermoepidérmicos de grosor parcial [IPP], colgajos locales de avance fasciocutáneo en V-Y, colgajos de rotación, cierre más injerto, colgajo más injerto, colgajo con sepultamiento de testículo con colgajos laterales e injerto y cierre por segunda intención con uso de baños de asiento con ácido bórico.

Se realizó un análisis estadístico con medidas de tendencia central y dispersión. Se realizó la prueba estadística de $\mathrm{T}$ de Student y se consideró significativo un valor de $\mathrm{p} \leq 0,05$.

Para la tabulación y presentación de los datos se utilizó el software MS Excel 2015.

\section{Resultados}

Durante el período de estudio se identificaron 43 pacientes a quienes se les realizó resección genitoperineal por secuelas del tratamiento de la gangrena de Fournier. De ellos $36(81,1 \%)$ de los casos eran hombres, con un promedio de edad de 59,1 años (rango de 17 a 86 años), y 7 (18,9\%) eran mujeres con un promedio de edad de 58,6 años (rango de 54 a 77 años). 12 pacientes fueron derivados desde el 
Hospital Luis Tisné Brousse, o el equipo de Cirugía Plástica del Hospital del Salvador fue explícitamente a realizar el procedimiento de cobertura en dicho establecimiento.

Del total de pacientes $72,7 \%$ presentaron diagnóstico de diabetes mellitus tipo 2, sin embargo, un $95,4 \%$ de los pacientes presentaron al menos una

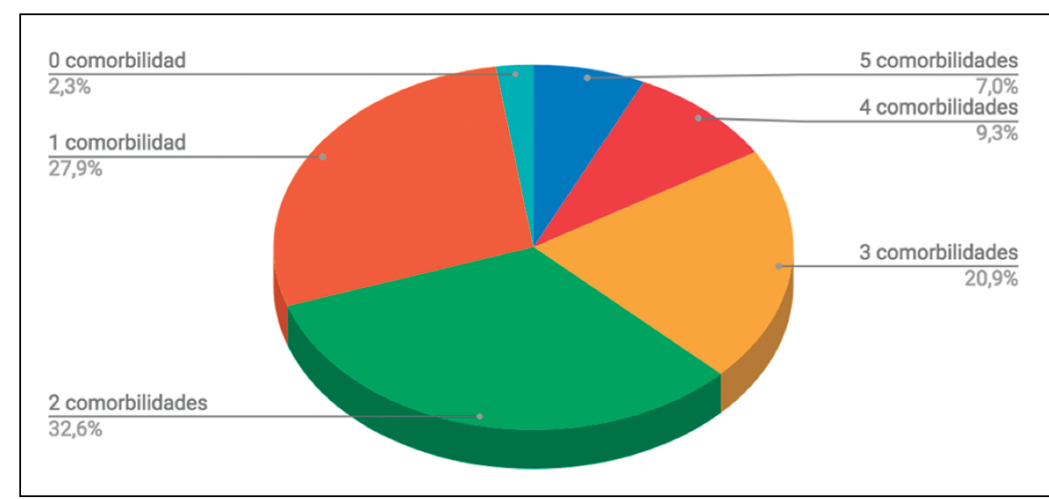

Figura 1. Distribución de pacientes según número de comorbilidades.

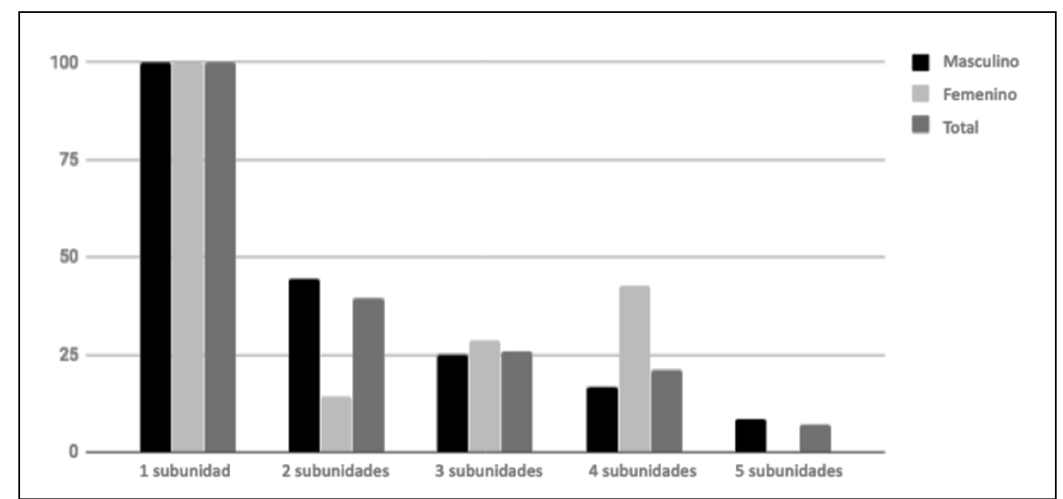

Figura 2. Distribución en porcentaje de las subunidades afectadas según sexo y total de pacientes.

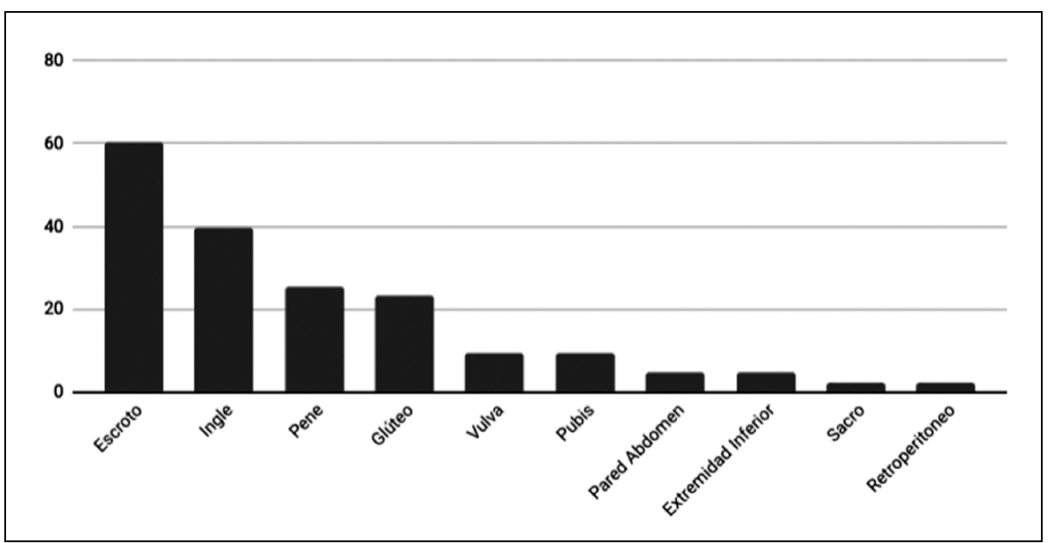

Figura 3. Porcentaje de subunidades afectadas posterior a la resección genitoperineal por gangrena de Fournier en el total de pacientes. comorbilidad de importancia (Figura 1), presentando la mayoría de los pacientes $(81,3 \%)$ entre 1 y 3 comorbilidades.

Respecto a las subunidades afectadas, por concepto la zona del periné estuvo afectada en el 100\% de los casos, las zonas restantes por frecuencia en el hombre fueron el escroto, ingle, pene, glúteo, pubis, extremidad inferior, abdomen y un paciente grave con compromiso de múltiples subunidades abarcando inclusive el retroperitoneo. En la mujer las subunidades afectadas de mayor a menor fueron; periné, glúteo, ingle, y casos aislados de compromiso de la zona pubiana, sacro y pared abdominal (Figura 2). Se grafica la distribución de las subunidades según porcentaje en el total de los pacientes en la Figura 3. El número de subunidades involucradas se asocia directa y significativa en relación al número de intervenciones quirúrgicas. Pacientes entre 1 a 3 subunidades afectadas requirieron en promedio 3,81 $\pm 1,84$ ( 27 pacientes) procedimientos quirúrgicos en comparación a los que presentaron 3 a 5 subunidades afectadas, con un promedio de cirugías de 6,25 $\pm 3,77$ (12 pacientes), siendo esto estadísticamente significativo $(\mathrm{p} \leq 0,05)$.

Siguiendo esta misma línea, los pacientes con dos subunidades afectadas $(\mathrm{n}=19)$, la mayoría fueron resueltos mediante cierre primario $(n=5 / 26,3 \%)$, seguido de IPP $(n=4 / 21,1 \%)$ y cierre parcial/IPP $(\mathrm{n}=3 / 15,8 \%)$. En caso de 3 subunidades afectadas $(\mathrm{n}=11)$ se resolvió con cierres parciales seguido de IPP en la mayoría de los casos $(n=4 / 36,4 \%)$

Finalmente, en caso de compromiso de 4 a 5 subunidades $(\mathrm{n}=12)$, gran parte de estos casos se resolvieron con colgajos $(n=5 / 41,7 \%)$.

Dependiendo de la zona afectada, generalmente, fue resuelta con cierre parcial seguido de IPP o cierre primario si la lesión estuvo localizada preferentemente en la zona del periné anterior con compromiso escrotal, cierre primario en los casos de defecto de cobertura de la zona inguinal. E IPP cuando la afectación involucró el pene. En caso de presencia de ano flotante, la mayoría de los pacientes presentaron entre 3 a 5 subunidades afectadas y fueron manejados con colgajos fasciocutáneos. En los pacientes que se utilizó Flexi-Seal ${ }^{\circledR}$ se pudo lograr selle hermético con $\mathrm{VAC}^{\circledR}$ sin problemas previo a la realización de los colgajos (Figura 4).

Una minoría de pacientes requirieron de penectomía, un caso $(2,35 \%)$ y orquiectomía $(4,65 \%)$ en dos.

La mayoría de los pacientes presentaron como origen topográfico de enfermedad el urológico en $44,4 \%$, seguido del anorrectal $(36,1 \%)$ y cutáneo $(19,4 \%)$. 


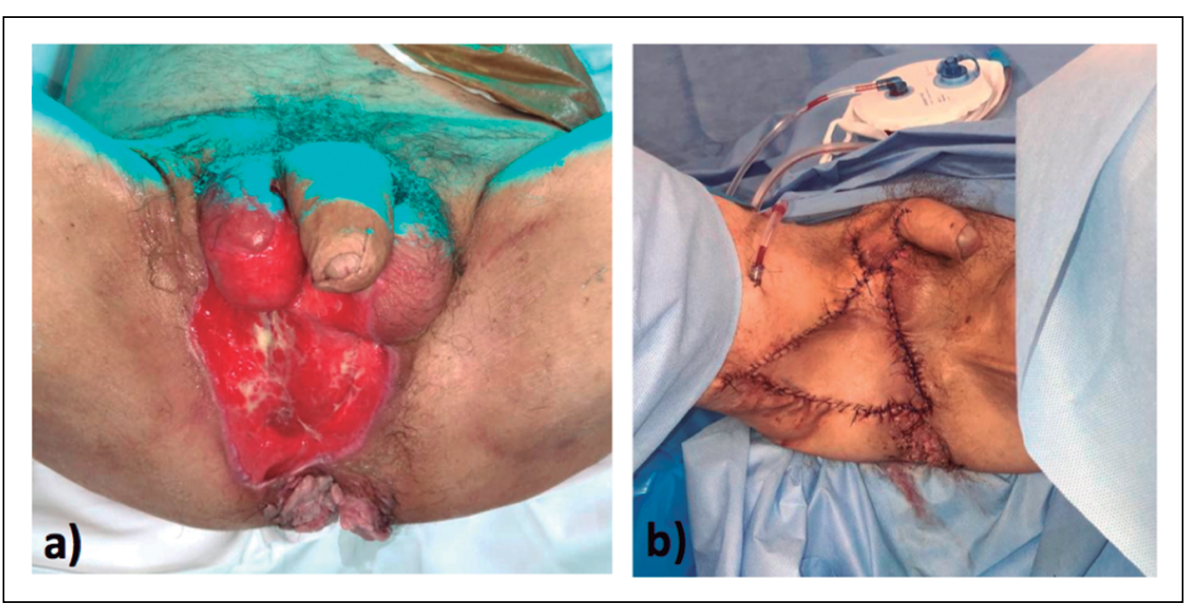

Figura 4. a) Paciente de 62 años con antecedentes de hipertensión arterial y artritis reumatoidea con ano flotante secundario a gangrena de Fournier por absceso anorrectal. Se observa uso de sistema de manejo fecal tipo Flexy-Seal ${ }^{\circledR}$ y sonda urinaria. b) Instalación de VAC ${ }^{\circledast}$ colocando espuma de poliuretano en defecto de cobertura y alrededor de sonda rectal y sonda urinaria para evitar pérdida de hermeticidad y lograr vacío. Ventosa ubicada craneal a la lesión.

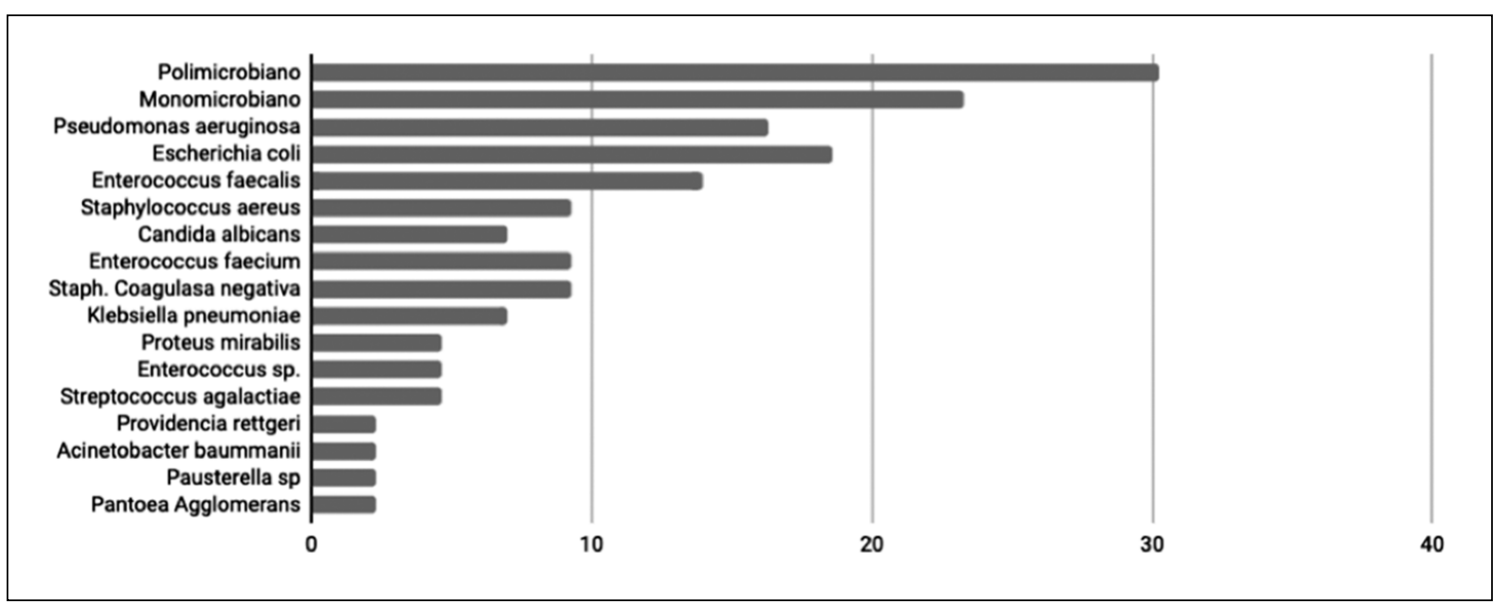

Gran parte de los cultivos de tejidos obtenidos luego de un aseo quirúrgico en pabellón fueron polimicrobianos. Las bacterias aisladas la mayoría correspondieron a Escherichia coli, seguido de Pseudomonas aeruginosa, Enterococcus faecalis y Staphylococcus aureus. La distribución porcentual de los patógenos aislados de los descritos y otros se resume en la Figura 5.

El uso de $\mathrm{VAC}^{\circledR}$ se utilizó en un $20,5 \%$ de los pacientes, quienes en promedio presentaron 3,25 subunidades afectadas.

El número de procedimientos quirúrgicos realizados en el total de pacientes, contabilizando aseos quirúrgicos, colostomías y reconstrucción genitoperineal, fue de 4,56 $\pm 2,7$, con un promedio de estadía hospitalaria de $49 \pm 25,5$ días.

Las técnicas más utilizadas para la reconstrucción de estos pacientes fueron: colgajos $(23 \%)$, cierre parcial más IPP (20\%), cierre primario (16\%) e injerto $(16 \%)$, cierre por segunda intención $(10 \%)$, colgajo más IPPF $(7 \%)$ y cierre parcial para cierre por segunda intención de zona restante $(5 \%)$. Finalmente, entre los colgajos más utilizados fueron los de avance fasciocutáneos en V-Y (Figuras 6 y 7). Especialmente, el grupo más desafiante fueron los que presentaron ano flotante ( 8 pacientes, $18,6 \%$ ) en quienes requirieron de reconstrucciones complejas mayoritariamente con colgajos.

La morbilidad posoperatoria fue de un $30,2 \%$ en el grupo manejado sólo con injerto; hubo un caso de pérdida total del injerto que requirió de nuevo injerto y otro con pérdida parcial que se manejó adecuadamente con curaciones para cierre por segunda intención. Al grupo que se le realizó cobertura sólo con cierre primario, la principal complicación fue dehiscencia parcial (4), manejado en la mitad de
Figura 5. Distribución porcentual de los distintos patógenos aislados en los cultivos de tejidos en pabellón. 


\section{ARTÍCULO ORIGINAL}

Figura 6. Tipos de cobertura realizados en la reconstrucción genitoperineal y subtipo de colgajos realizados.
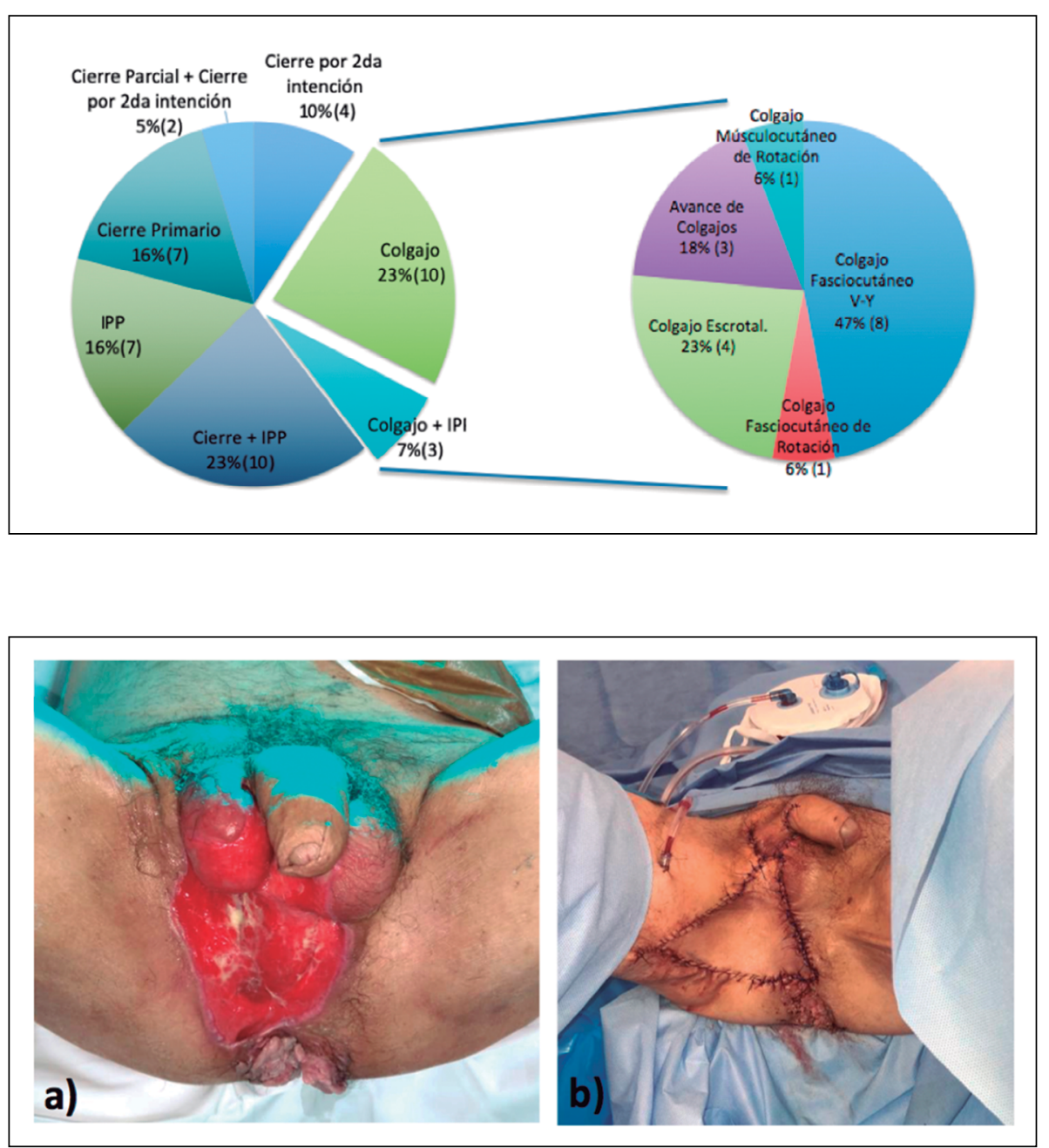

Figura 7. a) Paciente de sexo masculino de 49 años antecedentes de DM2 y condilomatosis perianal secundario a inmunodeficiencia por VIH. Defecto de cobertura de las subunidades periné anterior y escrotal de predominio derecho. b) Colgajo de avance fasciocutáneo en $\mathrm{V}$-Y de cara medial de muslo derecho y colgajo de avance escrotal logrando cobertura completa. los casos con cierre por segunda intención, seguido de un caso que requirió re-sutura y un último caso cierre más BiVAC (drenaje con hemovac a la cavidad, sutura de la dehiscencia y $\mathrm{VAC}^{\circledR}$ incisional). También en el grupo manejado sólo con colgajos, hubo dos casos de dehiscencia parcial, uno de ellos se manejó adecuadamente con nuevo cierre de la dehiscencia asociado a BiVAC y el otro con baños de asiento de ácido bórico, y por último, un caso de morbilidad mayor por contaminación con heces que requirió de aseo quirúrgico para luego reavance de colgajos para lograr nuevamente cobertura. En los casos de cierre parcial asociado a uso de injerto dermoepidérmico, la morbilidad principal (3 casos) fue de pérdida parcial del injerto que se manejó con cierre en cuña y con colgajos de avance locales, y el último caso se reinjertó con piel parcial. Finalmente, hubo un caso de morbilidad a largo plazo en el gru- po manejado mediante cierre por segunda intención por cicatriz retráctil en la base del pene.

Finalmente, englobando los procedimientos reconstructivos realizados en IPP, colgajos y cierres primarios, por lo tanto, contabilizando por separado los que recibieron cobertura combinada (ejemplo: colgajo + IPP). Obtuvimos que proporcionalmente hubo mayores complicaciones por IPP en un $25 \%$ principalmente por pérdida parcial, seguido de los colgajos mayoritariamente por dehiscencia (23\%) $\mathrm{y}$, por último, los cierres primarios con un $9,3 \%$ también por dehiscencia.

\section{Discusión}

La gangrena de Fournier se caracteriza por infección potencialmente mortal, necrotizante y rápidamente progresiva que involucra el periné, 
genitales externos, pudiendo diseminarse al largo de la fascia de Buck del pene, fascia perineal de Colles y extenderse hasta la fascia de Scarpa de la pared abdominal anterior ${ }^{7}$. Se entiende de esta forma la gran extensión que puede abarcar, desde muslos hasta el abdomen, significando una amplia resección de las distintas subunidades anatómicas que en ocasiones genera grandes defectos que resultan desafiantes para el cirujano reconstructivo para lograr una adecuada cobertura, especialmente en casos de ano flotante. Creemos que la descripción del defecto en relación a las subunidades involucradas es más adecuada que meramente el registro del tamaño del área ${ }^{12}$, ya que, a modo de ejemplo, una subunidad pequeña que abarque la zona perianal, frecuentemente es de mayor dificultad de cobertura en comparación a una gran lesión ubicada en zona inguinal, además, se comprueba que se asocia directamente y en forma significativa al número de procedimientos quirúrgicos requeridos. Siguiendo esta misma premisa, y a la luz de los resultados obtenidos, si nos encontramos con un defecto en la zona escrotal, lo más adecuado a nuestra opinión es intentar cierre primario o utilizar el mismo tejido escrotal remanente para cobertura con colgajo. La zona del pene y de preferencia en el cuerpo de este se resolvió sin mayores contratiempos con IPP. En la zona inguinal, incluso con compromiso parcial glúteo, es posible intentar un cierre primario. Para optimizar la cicatrización de esta zona se puede utilizar un $\mathrm{VAC}^{\circledR}$ de tipo incisional. Por último, en casos de ano flotante, en que la mayoría de nuestros pacientes presentaron varias subunidades afectadas, se resolvieron principalmente mediante colgajos fasciocutáneos.

Por otra parte, tomando datos numéricos y no de localización, es más probable lograr cobertura mediante un cierre primario o IPP en caso de compromiso de dos subunidades, por el contrario, en defectos de mayor envergadura con compromiso entre 4 a 5 subunidades, lo más probable es que requieran uso de colgajos fasciocutáneos o musculocutáneos para la cobertura.

Existe consenso en que un manejo agresivo y precoz $^{13}$ de estos pacientes mejora el pronóstico, lo cual condiciona en ocasiones la necesidad de realizar penectomía en un $15 \%$ y hasta un $30 \%$ de orquiectomía, porcentajes mayores a los reportados en nuestro estudio ${ }^{6}$. La enfermedad generalmente afecta predominantemente a hombres y rara vez mujeres ${ }^{1,7}$. Los factores predisponentes son múltiples, estos incluyen distintas condiciones tales como diabetes mellitus (predisposición más común ${ }^{3}$ ), hipertensión arterial, insuficiencia renal crónica, neoplasias malignas, alcoholismo crónico, inmunosupresión, trauma local, entre otras. Estas se asocian a respuesta inmunitaria celular reducida promoviendo la infección. Encontramos estas comorbilidades y otras, frecuentes en nuestros pacientes, presentando al menos una de ellas en el 97,7\%. La diabetes mellitus fue la más frecuente de todas, más del 70\% de nuestros pacientes, concordante con otros trabajos publicados que dan cifras entre $40 \%$ a $100 \%$ de los pacientes con gangrena de Fournier ${ }^{1,7}$.

La propagación de infecciones colorrectales (30\%-50\% de los casos), seguida del urogenital $(20 \%$ a $40 \%$ ) e infecciones cutáneas en un $20 \%$ son las principales ${ }^{3}$, siendo en nuestra serie en primer lugar el urológico. La propagación anorrectal es a consecuencia de abscesos colorrectales o perianales, instrumentación rectal, perforación del colon, trauma, etc. Los principales factores urogenitales son por extravasación urinaria, estenosis uretral, instrumentación, inflamación del tracto genitourinario y cirugías del pene y escroto. Por último, fuentes de infección de la piel que incluyen ulceración del escroto, hidrosadenitis supurativa, complicaciones de procedimientos quirúrgicos o serios traumas ${ }^{14}$.

En relación a los cultivos de tejidos obtenidos durante los aseos quirúrgicos en pabellón, la gran mayoría fue polimicrobiano, siendo el agente aislado en mayor frecuencia la Pseudomona aeruginosa seguido de la Escherichia coli, luego en menor proporción otras bacterias Gram positivas, anaerobios e incluso fúngicas, concordante con otros trabajos extranjeros y nacionales ${ }^{13,15,16}$.

La elección de reconstrucción se basa en las características del defecto, es decir, el tamaño, la ubicación y profundidad, así como la disponibilidad de tejido local ${ }^{17}$. La subunidad más afectada después de la perineal fue la zona escrotal, por lo tanto, conocer su anatomía junto a técnica reconstructiva proporciona excelentes resultados clínicos y estéticos ${ }^{17}$.

Otro método utilizado es el IPP cuando persiste un defecto a pesar de intentar un cierre primario o posterior a un avance de colgajo incompleto; resultó ser satisfactorio en la mayoría de los casos, pero hay que considerar los riesgos de contaminación y pérdida del injerto en forma parcial o completa, especialmente si se utiliza en cercanía de la zona perianal. Presenta la desventaja de ser más vulnerable respecto al uso de un colgajo fasciocutáneo o musculocutáneo y los riesgos inherentes a la contracción secundaria. Resulta ser útil tanto para los defectos de la zona perineal como para cobertura escrotal. El uso de VAC ${ }^{\circledR}$ resulta ser de utilidad para proteger y aumentar la probabilidad de prendimiento del injerto ${ }^{18}$. 
El registro de mortalidad no está presente ya que mucho de los pacientes fueron ingresados y manejados por distintos equipos, de los cuales, una vez resuelto el cuadro agudo crítico y estabilización de los pacientes, fueron derivados al equipo de cirugía plástica para continuar con los aseos quirúrgicos y planificación de cobertura. Durante esta etapa no hubo ningún caso de mortalidad.

Respecto a la morbilidad global posterior a reconstrucción varía en la literatura entre $9 \%{ }^{12}$ y $40 \%{ }^{7}$, concentrándose mayoritariamente en la pérdida parcial de IPP, seguido de dehiscencia parcial de los colgados y necrosis parcial de los mismos, similar en lo reportado en nuestro trabajo. Por lo tanto, considerar en lo posible como primera alternativa el uso de colgajos locales, tales como escrotales o fasciocutáneos. En caso de necesidad de resolver un defecto de cobertura importante, considerar colgajos musculares o musculocutáneos.

Consideramos que, si es posible lograr una cobertura con cierre primario, siempre y cuando sea libre libre de tensión, debe ser una opción preferente. De lo contrario considerar los colgajos fasciocutáneos de avance o de rotación. Estos han resultado versátiles para la cobertura de la mayoría de los defectos, inclusive en algunos casos con exposición de testes en que se optó por sepultamiento del/los testículo/s con colgajos laterales. En defectos profundos que requiera de eliminar espacio muerto importante, se debe considerar colgajos musculares o musculocutáneos. En casos de presentar tejido escrotal, este se debe considerar como colgajo ya que presenta la ventaja de ser elástico y adecuado para la cobertura de zonas adyacentes. En caso de exposición de testes sin cobertura adecuada con colgajo escrotal, se puede optar con IPP. También se debe considerar el IPP en caso de cobertura incompleta en otros tipos de colgajos. Tal como fue mencionado anteriormente, el prendimiento del IPP en esta zona resulta de gran dificultad tanto por la anatomía, pliegues, movilidad excesiva y riesgo de contaminación el cual puede ser mitigado con la aplicación de $\mathrm{VAC}^{\circledR}$. El cierre por segunda intención no se debe considerar como primera posibilidad a no ser que por condición global de paciente y riesgos quirúrgicos contraindiquen realización de procedimientos quirúrgicos en pabellón.

El uso de Flexi-Seal ${ }^{\circledR}$ en comparación a la colostomía nos parece costo-efectivo ya que elimina el procedimiento quirúrgico de colostomía y reconstrucción de tránsito, además, de contribuir en el confort del paciente ya que se limita la derivación fecal al periodo comprendido de reconstrucción genitoperineal y elimina los riesgos atribuidos a la colostomía $^{19}$ y de la reconstitución de tránsito. Hay que considerar que muchos de los pacientes colostomizados por este motivo, dada las características de estos pacientes con sus múltiples comorbilidades, no se les realiza nunca la reconstitución.

El uso $\mathrm{VAC}^{\circledR}$ en los procesos previo a la cobertura es factible y conveniente, es más, permite inducir más rápidamente tejido de granulación por medio del incremento de la perfusión local, migración de fibroblastos y eliminación del exceso de exudado contribuyendo, por tanto, en lograr un lecho adecuado de cobertura en un plazo menor ${ }^{9}$. Este puede ser instalado incluso en localizaciones difíciles, logrando adecuado selle hermético incluyendo dispositivos para manejo fecal como lo es el Flexi-Seal ${ }^{\circledR}$ o sonda urinaria. Para lograr ello, se debe procurar trabajar en piel libre de folículos pilosos, proteger la piel con una película protectora tipo Cavilon $^{\circledR}(3 \mathrm{M})$ u OpSite (Smith \& Nephew) en spray para lograr adherencia adecuada del apósito transparente tipo Tegaderm ${ }^{\circledR}$ en piel sana alrededor del defecto a cubrir, además, este apósito protegerá de la piel de la espuma si este por algún motivo deba quedar por sobre la piel. La espuma en contacto directo del defecto debe ser del mismo tamaño o marginalmente menor para que durante el vacío los márgenes de la lesión tiendan a contraerse. En el defecto alrededor del ano flotante, colocar una espuma en forma de donas para lograr el paso del Flexi-Seal ${ }^{\circledR}$ a través de este. Se debe sellar todo el sistema con otros apósitos transparentes, teniendo especialmente cuidado de sellar con esto el Flexi-Seal ${ }^{\circledR}$ a la donas de espuma de poliuretano. De esta forma permitirá un sello hermético adecuado, con los beneficios del sistema de cierre asistido al vacío y al mismo tiempo eliminación de las heces por medio de la sonda rectal.

Finalmente, podemos concluir que la reconstrucción genitoperineal en pacientes con gangrena de Fournier resulta ser un desafío para el cirujano plástico reconstructivo. Requiere de un manejo precoz y agresivo con resección amplia de la zona involucrada. Se describe en detalle las características de los pacientes y del defecto de cobertura en subunidades anatómicas que se relacionan directamente con el número de procedimientos quirúrgicos. El conocimiento amplio de opciones de reconstrucción que van desde cierre por segunda intención, cierre primario, uso de injertos dermoepidérmicos de grosor parcial y el conocimiento de las técnicas de colgajos de avance y rotación fasciocutáneos, son herramientas importantes y necesarias para manejar este tipo de pacientes. El uso de mecanismo de derivación fecal mediante el uso de Flexi-Seal ${ }^{\circledR}$ o colostomías, resultan medidas adecuadas especialmente en casos de ano flotante. 
Tips

1. La gangrena de Fournier (GF) es una forma rápidamente progresiva y potencialmente letal de fasceitis necrotizante de las regiones perineal, genital o perianal.

2. El reconocimiento temprano junto con una extensa resección del tejido necrótico, tratamiento antimicrobiano y volemización agresiva, son el pilar del manejo de la gangrena de Fournier.

3. La elección de reconstrucción se basa en las características del defecto: el tamaño, la ubicación y profundidad, así como la disponibilidad de tejido local.

4. Las técnicas de reconstrucción utilizadas son: cierre primario, IPP, colgajos, la combinación de las anteriores $\mathrm{y}$, por último, cierre por segunda intención.

6. Más del $95 \%$ de los pacientes presentan al menos una comorbilidad y de ésta la más frecuente es diabetes mellitus tipo II.

7. La descripción del defecto en relación a las subunidades involucradas es más adecuado que el registro del tamaño del área.

\section{Responsabilidades éticas}

Protección de personas y animales. Los autores declaran que para esta investigación no se han realizado experimentos en seres humanos ni en animales.

Confidencialidad de los datos. Los autores declaran que en este artículo no aparecen datos de pacientes.

Conflictos de interés: no hay.

\section{Bibliografía}

1. Wróblewska M, Kuzaka B

Borkowski T, Kuzaka P, Kawecki D, Radziszewski P. Fournier's gangrenecurrent concepts. Pol J Microbiol. 2014;63:267-73.

2. Castillo F, Moraga J, Pérez P, Álvarez C, Iglesias A. Diagnóstico y manejo precoz de la gangrena de Fournier. Rev Chil Cir. 2015;67:181-4.

3. Chennamsetty A, Khourdaji I, Burks F, Killinger KA. Contemporary diagnosis and management of Fournier's gangrene. Ther Adv Urol. 2015;7:203-15. doi: $10.1177 / 1756287215584740$

4. Lin CT, Chang SC, Chen SG, Tzeng YS. Reconstruction of perineoscrotal defects in Fournier's gangrene with pedicle anterolateral thigh perforator flap. ANZ J Surg. 2016;86:1052-5.

5. Fournier JA. Gangrene foudroyante de la verge. Sem Med. 1883;3:345-8

6. Meki CS, Mangwiro TI, Lazarus J. Fournier's gangrene: outcome analysis and prognostic factors. S Afr J Surg. 2018;56:43-6.

7. Louro JM, Albano M, Baltazar J, Vaz M, Diogo C, Ramos S, Cabral L. Fournier's Gangrene: 10-Year Experience of a Plastic Surgery and Burns Department at a Tertiary Hospital. Acta Med Port. 2019;32:368-74. doi: 10.20344/ amp. 11003 .

8. Clayton M, Fowler J, Sharif R. Causes, presentation and survival of 57 patients with necrotizing fascitis of the male genitalia. Surg Gynaecol Obstet. 1990;170:49-55.

9. Ozkan OF, Koksal N, Altinli E, Celik A, Uzun MA, C1kman O. Fournier's gangrene current approaches. Int Wound J. 2016;13:713-6.

10. Mallikarjuna MN, Vijayakumar A, Patil VS, Shivswamy BS. Fournier's Gangrene: Current Practices. ISRN Surg. 2012;94:2437.

11. Ferreira P, Reis J, Amarante J, Silva A, Pinho C, Oliveira I, da Silva P. Fournier Gangrene: A review of 43 reconstructive cases. Plast Reconstr Surg.2007;119:17584.

12. Chen SY, Fu JP, Wang CH, Lee TP, Chen $\mathrm{SG}$. Fournier gangrene: a review of 41 patients and strategies for reconstruction. Ann Plast Surg. 2010;64:765-9.

13. Lin TY, Cheng IH, Ou CH, Tsai YS, Tong YC, Cheng HL. Incorporating Simplified Fournier's Gangrene Severity Index with early surgical intervention can maximize survival in high-risk Fournier's gangrene patients. Int J Urol. 2019;26:737-43. doi: 10.1111/iju.13989.

14. Wang L, Han X, Liu M, Mei L, Ma Y, Li B. Experience in management of Fournier's gangrene: a report of 24 cases. J Huazhong Univ Sci Technolog Med Sci. 2012;32:719-23. doi: 10.1007/s11596012-1024-4.

15. Yilmazlar T, Gulcu B, Isik O, Ozturk E. Microbiological aspects of Fournier's gangrene. Int J Surg. 2017;40:135-8. doi: 10.1016/j.ijsu.2017.02.067. Epub 2017 Feb 28.
16. Castillo F, Moraga J, Pérez P, Álvarez Z, Iglesias A. Diagnóstico y manejo precoz de la gangrena de Fournier. Rev Chil Cir. 2015;67:181-4

17. Singh A, Ahmed K, Aydin A, Khan MS, Dasgupta P. Fournier's gangrene. A clinical review. Arch Ital Urol Androl. 2016;88:157-64. doi: 10.4081/ aiua.2016.3.157.

18. Seung Bum Pyo, Kun Yong Sung, Hong Sil Joo, Jin Kyung Song, Seong Yoon Lim. Vaccum-Assisted Closure Therapy in Split-Thickness Skin Graft on the Wound on the Contours of the Body. J Wound Manag Res. 2017;13:35-9.

19. Oguz A, Gümüş M, Turkoglu A, Bozdag Z, Ülger BV, Agacayak E. Fournier's Gangrene: A Summary of 10 Years of Clinical Experience. Int Surg. 2015;100:934-41. doi: 10.9738/ INTSURG-D-15-00036.1. Epub 2015 Apr 10.

20. Abcarian H, Eftaiha M. Floating freestanding anus. A complication of massive anorectal infection. Dis Colon Rectum 1983;26:516-21.

21. Ozkan OF, Altýnlý E, Koksal N, Celik A, Uzun MA, Cikman O. Combining FlexiSeal and negative pressure wound therapy for wound management in Fournier's gangrene. Int Wound J. 2015;12:364-5. doi: 10.1111/iwj.12103.

22. Hallock GG. The Development of the Medial Circumflex Femoral Artery Perforator (MCFAP) Flap. Semin Plast Surg. 2006;20:121-6. doi: 10.1055/s2006-941719. 